\title{
Nonadditivity of the Adsorption Energies of Linear Acenes on Au(111): Molecular Anisotropy and Many-Body Effects
}

\author{
Friedrich Maass, ${ }^{\dagger}$ Mohsen Ajdari, ${ }^{\dagger}$ Fairoja Cheenicode Kabeer, ${ }^{\ddagger}{ }^{\circ}$ Maximilian Vogtland, ${ }^{\dagger}$

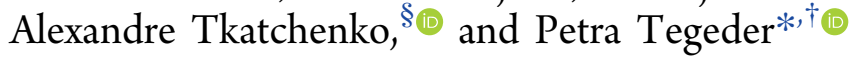 \\ ${ }^{\dagger}$ Ruprecht-Karls-Universität Heidelberg, Physikalisch-Chemisches Institut, Im Neuenheimer Feld 253, 69120 Heidelberg, Germany \\ ${ }^{\ddagger}$ Fritz Haber Institute of the Max Planck Society, Faradayweg 4-6, 14195 Berlin, Germany \\ ${ }^{\S}$ Physics and Materials Science Research Unit, University of Luxembourg, L-1511 Luxembourg, Luxembourg
}

Supporting Information

\begin{abstract}
Adsorption energies of chemisorbed molecules on inorganic solids usually scale linearly with molecular size and are well described by additive scaling laws. However, much less is known about scaling laws for physisorbed molecules. Our temperature-programmed desorption experiments demonstrate that the adsorption energy of acenes (benzene to pentacene) on the $\mathrm{Au}(111)$ surface in the limit of low coverage is highly nonadditive with respect to the molecular size. For pentacene, the deviation from an additive scaling of the adsorption energy amounts to as much as 0.7 $\mathrm{eV}$. Our first-principles calculations explain the observed nonadditive behavior in terms of anisotropy of molecular polarization stemming from many-body electronic correlations. The observed nonadditivity of the adsorption energy has implications for surface-mediated intermolecular interactions and the ensuing on-surface self-assembly.

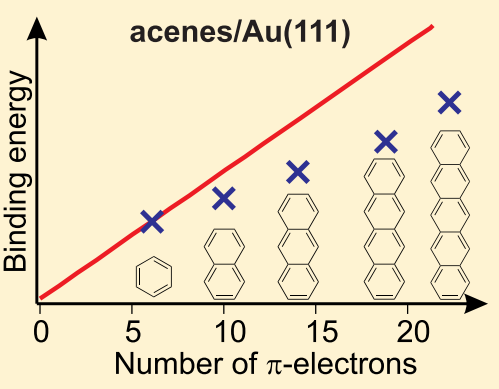
Thus, future coverage-dependent studies should aim to gain insights into the impact of these complex interactions on the selfassembly of $\pi$-conjugated organic molecules on metal surfaces.
\end{abstract}

$\mathrm{F}$ or $\pi$-conjugated organic molecules adsorbed on metallic substrates, the binding mainly arises from the $\pi$-electrons interacting with the metal surface and accordingly the binding strength strongly depends on the size of the $\pi$-electron system. For chemisorbed molecules, e.g., benzene and naphthalene on $\mathrm{Pt}(111)$, it is widely accepted that the adsorption energy is additive and scales linearly with the size of the $\pi$-electron system. $^{1-5}$ However, for physisorbed molecules no clear picture exists about the scaling of the binding energy with the extent of the electronic system. ${ }^{6}$ Consequently, investigating the effects of the size of the $\pi$-electron system on the binding strength of physisorbed compounds on a metal surface allows the much needed benchmarks for metal $-\pi$ interactions to be obtained.

The benzene/ $\mathrm{Au}(111)$ system has been investigated in detail, ${ }^{4,7}$ and recently we precisely established the binding energy of benzene on $\mathrm{Au}(111)$ and also on the other (111)oriented coinage metal surfaces by a combined experimental and theoretical study. ${ }^{8,9}$ In contrast, for the larger acenes adsorbed on (111)-coinage metals only for some molecular systems binding energies have been determined ${ }^{10-13}$ from rather unprecise methods, such as the Redhead approximation. ${ }^{14}$ In addition, van der Waals (vdW) interactions have been included at a rather low level in available DFT $+v d W$ calculations; thus, reliable adsorption energy values for acenes on metals are not yet available. ${ }^{15,16}$

Here, we systematically study the binding properties of naphthalene, anthracene, tetracene, and pentacene on $\mathrm{Au}(111)$ by means of detailed coverage dependent temperature- programmed desorption (TPD) measurements and by applying the so-called complete analysis ${ }^{17,18}$ in combination with recently developed first-principles calculations that account for the nonlocality of electron correlation. ${ }^{19}$ Both our experiments and calculations yield a strongly nonadditive adsorption energy as a function of the acene length. We attribute this nonadditive effect to the anisotropic polarization of the acenes stemming from electronic many-body correlations.

Figure 1 shows prototypical TPD spectra for acenes of varying size on $\mathrm{Au}(111)$. One can observe a shift toward higher desorption temperatures with rising number of phenyl rings ( $\pi$-electrons), in agreement with ref 10 . Specifically, for all acenes a low-temperature desorption peak is observed, which can be associated with desorption from the second or higher layers (multilayers). The desorption features at higher temperatures can be assigned to desorption from the monolayer $(\mathrm{ML})$, i.e., molecules in direct contact with the metal substrate, which are more strongly bound compared to molecules above the monolayer. In the case of benzene, naphthalene, and pentacene, a third desorption peak is found. We attribute this to desorption from a more densely packed compressed phase, as reported for other aromatic molecules on noble metal surfaces. ${ }^{20-27}$ Note that the temperature range in

Received: January 29, 2019

Accepted: February 15, 2019

Published: February 15, 2019 


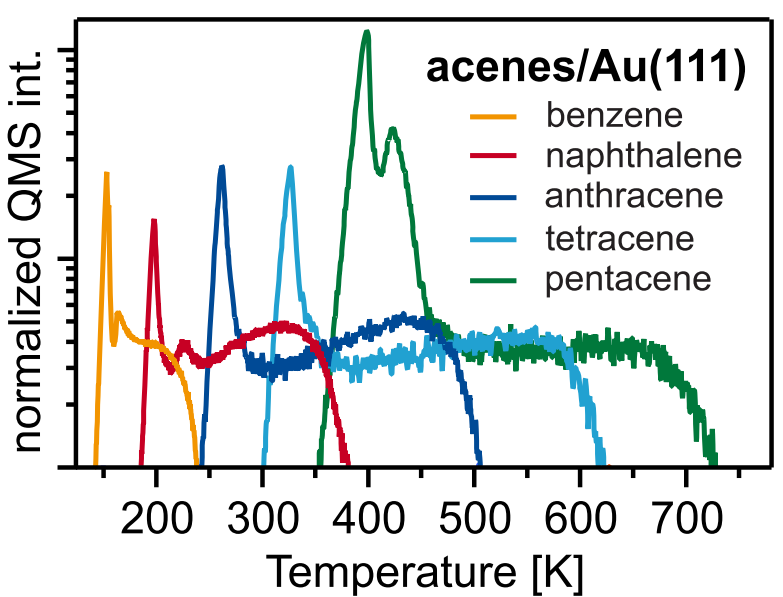

Figure 1. Prototypical TPD spectra for acenes adsorbed on $\mathrm{Au}(111)$.

which monolayer desorption occurs increases with increasing number of phenyl rings.

To precisely analyze the adsorption properties of acenes on $\mathrm{Au}(111)$, we determined the binding energies of naphthalene, anthracene, tetracene, and pentacene as a function of coverage by using the so-called complete analysis method. ${ }^{17}$ This method has the advantage that no guess for the pre-exponential factor $(\nu)$ has to be made. This is of particular importance because the value of this factor depends on a number of system parameters such as the size of the molecule and its vibrational degrees of freedom, among other contributions. ${ }^{28}$ Furthermore, it is the only method which allows analyzing measurements to determine the coverage dependency of $\nu$ and the desorption energy $\left(E_{\text {Des }}\right){ }^{18}$ We already successfully used this method to elucidate the binding energies of benzene on the coinage metal surfaces $\mathrm{Au}(111), \operatorname{Ag}(111)$, and $\mathrm{Cu}(111)^{8,9}$ as well as azobenzenes on noble metals. ${ }^{21,29,30}$ To apply the complete analysis, TPD measurements over a wide range of initial coverages in the submonolayer regime have to be carried out, as exemplarily displayed in Figure 2a for pentacene $/ \mathrm{Au}(111)$. We defined a monolayer to the desorption spectrum shown in black. The integral of this spectrum is used as a reference to determine the coverage of all other TPD spectra shown in Figure 2a. While the falling edges at the high temperature cutoff of the monolayer to submonolayer desorption peaks lie on top of each other, the peak maxima strongly shift to lower temperatures with increasing coverage. This is a clear indication for repulsive lateral and substrate-mediated interactions. ${ }^{8,9,21,31}$ By applying the complete analysis evaluation routine, the binding energy as a function of coverage can be determined, which is displayed in Figure $2 \mathrm{~b}$. The data are fitted with a second-order polynomial fit resulting in a desorption energy in the limit of vanishing coverage (the intercept with the $y$-axis) of $E_{\text {Des }}(\theta \rightarrow 0 \mathrm{ML})=$ $1.80 \pm 0.15 \mathrm{eV}$. For comparison with theoretical data, this experimental value measured at finite temperatures has to be corrected to $E_{\text {Des }}$ at $0 \mathrm{~K}$ by adding $3 / 2 k_{\mathrm{B}} T_{\text {Des }}(\approx 0.03 \mathrm{eV})$, yielding a value of $1.83 \pm 0.15 \mathrm{eV}$. For the other acenes, we also analyzed the binding properties as a function of coverage using the complete analysis (see Supporting Information (SI)); the binding energy values for vanishing coverage are summarized in Figure 3 and Table 1. As can be clearly seen, the binding energy scales nonadditively with the number of $\pi$ electrons. To rationalize these experimental findings, in the following we present and discuss our first-principles calcu-
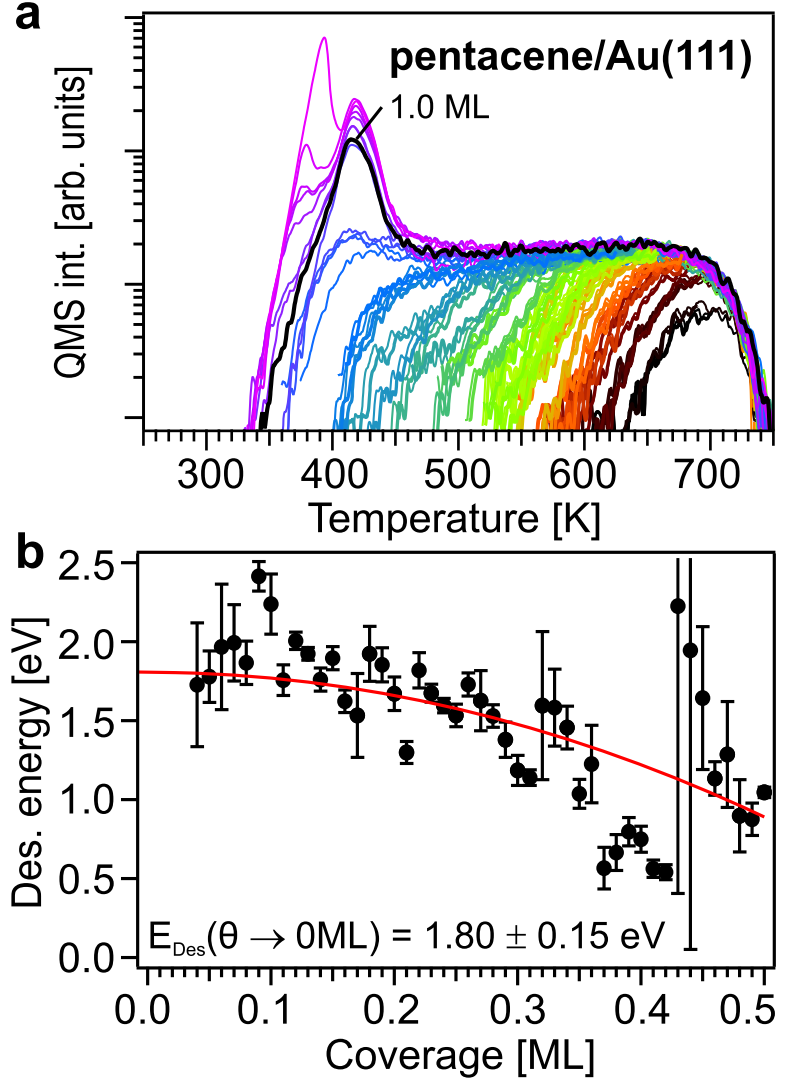

Figure 2. (a) Coverage dependent TPD spectra of pentacene adsorbed on $\mathrm{Au}(111)$ in the low coverage regime. TPD curve shown in black is assigned to a monolayer (ML) coverage. (b) Desorption energy as a function of pentacene coverage determined by applying the complete analysis. The red solid line represents a fit using a secondorder polynomial function to describe the experimental data.

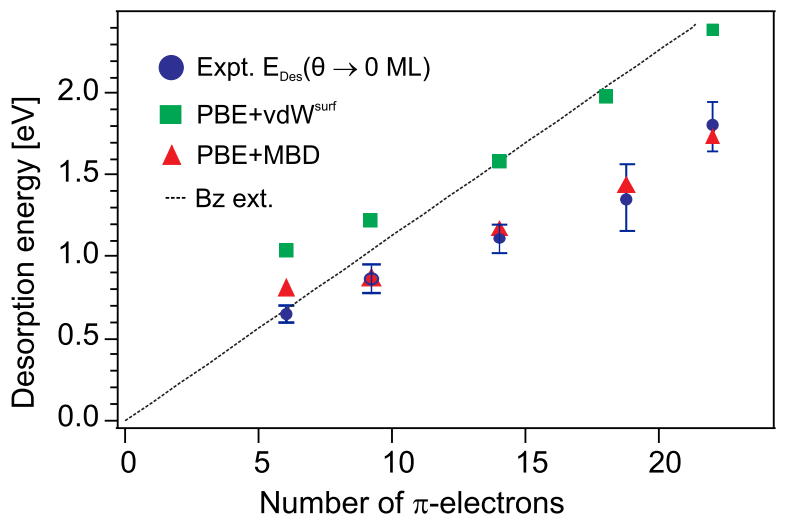

Figure 3. Desorption energy in the limit of vanishing coverage for the acenes, i.e., benzene, naphthalene, anthracene, tetracene, and pentacene on $\mathrm{Au}(111)$. The value for benzene $(\mathrm{Bz}) / \mathrm{Au}(111)$ determined via the complete analysis of TPD data has been adopted from ref. ${ }^{9}$ Computed binding energies for the acenes on $\mathrm{Au}(111)$ at the most preferable sites using $\mathrm{PBE}+\mathrm{vdW} \mathrm{W}^{\text {surf }}$ and $\mathrm{PBE}+\mathrm{MBD}$ calculations. The dashed line assumes a simple additive behavior of the adsorption energy per $\pi$-electron based on the measured $E_{D e s}$ for benzene.

lations that account for nonlocality of electronic correlation effects.

We have carried out DFT+vdW calculations for the adsorption of all studied acenes (from benzene to pentacene) 
Table 1. Computed Binding Energies (in eV) for the Acenes on $\mathrm{Au}(111)^{a}$

\begin{tabular}{|c|c|c|c|c|c|}
\hline & benzene & naphthalene & anthracene & tetracene & pentacene \\
\hline$P B E+v d W^{\text {surf }}$ & 1.00 & 1.19 & 1.59 & 1.99 & 2.39 \\
\hline $\mathrm{PBE}+\mathrm{MBD}$ & 0.80 & 0.88 & 1.16 & 1.44 & 1.73 \\
\hline Exp. values & $0.71 \pm 0.03^{b}$ & $0.91 \pm 0.1$ & $1.14 \pm 0.1$ & $1.38 \pm 0.2$ & $1.83 \pm 0.15$ \\
\hline
\end{tabular}

${ }^{a}$ All experimental values are corrected to $E_{\text {Des }}$ at $0 \mathrm{~K}$ and correspond to the limit of zero coverage (see text). ${ }^{b}$ The binding energy value has been adopted from ref 9 .

on $\mathrm{Au}(111)$. We employ a relatively large $(9 \times 5)$ supercell of $\mathrm{Au}(111)$ that allows comparison to low-coverage experiments, since lateral interactions in the $(9 \times 5)$ cell are negligible. In order to explore the infuence of different treatments of vdW interactions, we have employed two DFT+vdW methods, namely PBE+vdW ${ }^{\text {surf32 }}$ and PBE+MBD. ${ }^{19,33}$ The comparison between these two sets of calculations allows us to assess the potential relevance of many-body correlations (MBD) beyond the pairwise approximation ( $\left.\mathrm{vdW}^{\text {surf }}\right)$. As can be observed in Figure 3, the $\mathrm{PBE}+\mathrm{vdW} \mathrm{W}^{\text {surf }}$ calculations predict that the adsorption energy of acenes increases in an additive linear fashion, as expected from a simple atom-pairwise approximation for the vdW interactions, but in strong contrast to experimental observations. We stress that any pairwise approximation to $\mathrm{vdW}$ interactions would yield a simple additive scaling of the adsorption energy. Conversely, PBE $+\mathrm{MBD}$ calculations yield a pronounced nonadditive behavior of the adsorption energy as a function of acene size, and the $\mathrm{PBE}+\mathrm{MBD}$ adsorption energies turn out to be in excellent agreement with experiment (see Figure 3 and Table 1). The fraction of the adsorption energy induced by many-body electronic correlations strongly increases with molecular size, growing from $0.2 \mathrm{eV}$ for benzene to almost $0.7 \mathrm{eV}$ for pentacene.

The observed nonadditive behavior of the adsorption energy can be understood in terms of anisotropy of molecular polarization which stems from many-body electronic correlations. Already in the gas phase, the polarizability of acenes is strongly anisotropic. For example, the perpendicular component of the polarizability of benzene is twice smaller than the in-plane component. ${ }^{8}$ The anisotropy keeps growing with molecular size. ${ }^{34}$ Upon adsorption on a metal surface, the polarizability of the combined molecule/surface system can strongly deviate from the sum of polarizabilities of the isolated molecule and pristine surface. We already found in ref 8 that for the benzene/ $\mathrm{Au}(111)$ system the perpendicular component of the polarizability grows, while the in-plane component decreases, upon adsorption. This change in polarizability components results in less effective coupling between the inplane fluctuations of the electron density of the benzene molecule and the surface plasmons, leading to a concomitantly smaller vdW attraction in $\mathrm{PBE}+\mathrm{MBD}$ calculations compared to the PBE+vdW ${ }^{\text {surf }}$ method. A similar mechanism is in play for larger acene molecules, with even larger (de)polarization than that found for benzene $/ \mathrm{Au}(111)$. For the pentacene molecule on $\mathrm{Au}(111)$, we observe that the perpendicular $(z z)$ component of the polarizability grows by $11 \mathrm{bohr}^{3}$, while the in-plane $(x x$ and $y y)$ components are reduced by 7.2 and 4.0 bohr $^{3}$, respectively, compared to the separated molecule and surface. The decrease of the in-plane polarizability, which increases with acene size, explains the reduction and nonadditivity of acene adsorption energies compared to a simple additive scaling predicted by pairwise additive vdW models.
The presented calculations rely on several approximations whose accuracy we will briefly discuss here (see Methods section for more information). First, we used static unrelaxed structures for the acene molecules adsorbed at an equilibrium vertical position. For benzene $/ \mathrm{Au}(111)$, the change in the adsorption energy due to full geometry relaxation was found to be $0.02 \mathrm{eV}^{35}$ hence, it is completely negligible compared to the energies in Table 1. Second, at the desorption temperatures in the low coverage limit the molecules are mobile and hence there is no unique adsorption site. Thus, the cleanest comparison of experiments and calculations at low coverage is done by assuming an unreconstructed $\mathrm{Au}(111)$ surface.

When the experimental and theoretical results are merged, both elucidate the appreciable nonadditivity of the binding energy with the size of the $\pi$-electron system. As can be seen in Figure 3 and from Table 1, our calculated binding energies using the first-principles $\mathrm{PBE}+\mathrm{MBD}$ method are in excellent agreement with the experimentally determined values. Thus, we conclude that the nonadditive behavior can be explained by the anisotropy of the molecular polarization originated from many-body electronic correlations. The nonadditivity identified for the single molecules may also affect neighboring adsorption sites and thus influence the binding energies at higher coverages in the submonolayer regime. The TPD data clearly reveal the existence of repulsive lateral and substratemediated interactions in the submonolayer regime. Moreover the temperature range in which monolayer desorption occurs rises significantly with increasing molecular size, providing strong motivation for further studies at higher coverages.

In summary, we employed temperature-programmed desorption and first-principles calculations to precisely determine the binding strength of the acenes (benzene to pentacene) on the $\mathrm{Au}(111)$ surface. Contrary to chemisorbed molecules on metal surfaces, for which the adsorption energy scales additively with the size of the $\pi$-electron system, we found a strong nonadditivity for physisorbed molecules in the limit of vanishing coverage (single molecule) by both theory and experiment. Our PBE+MBD calculations that account for many-body correlations resulted in excellent agreement with the experimentally determined binding energies. The nonadditive behavior of the adsorption energy was attributed to the anisotropy of the acenes molecular polarization stemming from electronic many-body correlations. We anticipate that the identified nonadditive behavior of the binding energies will also have an impact on substrate-mediated intermolecular interactions and accordingly on self-assembly processes at surfaces at higher coverages in the submonolayer regime. Hence, future work should focus on coverage-dependent studies to elucidate substrate-mediated intermolecular interactions. 


\section{EXPERIMENTAL AND COMPUTATIONAL METHODS}

Experimental Methods. All experiments were performed under ultrahigh vacuum conditions at a base pressure of $1 \times 10^{-10}$ mbar. The crystals were mounted onto a liquid nitrogen cooled cryostat, and together with resistive heating a temperature range (measured directly at the substrate via a thermocouple) between 100 and $800 \mathrm{~K}$ was achievable and precisely controllable. Crystals were prepared by a standard cleaning procedure including $\mathrm{Ar}^{+}$sputtering and subsequent annealing to $750 \mathrm{~K}$. The respective molecules were evaporated from an effusion cell (anthracene, tetracene, and pentacene) or via a leak valve (naphthalene) and deposited on a $\mathrm{Au}(111)$ single crystal (the evaporation and sample temperatures for all investigated molecules can be found in the SI). To record TPD spectra, the samples were heated with a constant heating rate of $\beta=1 \mathrm{~K} / \mathrm{s}$ and the desorbing acences were monitored with a quadrupole mass spectrometer. The complete analysis method, which has been applied to analyze the coverage dependent TPD data, has been described in detail in ref 9 .

Computational Methods. For all DFT calculations, we used the all-electron/full-potential electronic-structure code FHIaims. $^{36,37}$ The $\mathrm{PBE}^{38}$ exchange-correlation (XC) functional and "light" computational settings were used for the calculations. The electron density was converged to $10^{-5} e$, and the total energy, to $10^{-6} \mathrm{eV}$. Relativistic effects were included via the atomic scalar zeroth-order regular approximation. ${ }^{39}$ The electronic levels around the conduction level were broadened with a Gaussian function with a width of 0.1 $\mathrm{eV}$. The total energies were calculated for zero-broadening based on the entropy of the electron gas. We have calculated the binding energies for acene molecules adsorbed on the $\mathrm{Au}(111)$ surface using the single-point calculations with both the $\mathrm{PBE}+\mathrm{vdW}$ surf32,40 and $\mathrm{PBE}+\mathrm{MBD}^{19}$ methods. The surface slab was modeled with a $9 \times 5$ supercell having four metallic layers, and a vacuum space of $40 \AA$ was included perpendicular to the surface to avoid the interaction between periodic images. The acene molecules were placed $3.08 \AA$ above the topmost $\mathrm{Au}$ layer of the slab. The difference in adsorption energetics was studied locating the acene molecules on the fcc site and bridge site of the Au slab. We used a Monkhorst-Pack grid of $2 \times 4 \times 1$ k-points.

The adsorption energies were calculated using

$$
E_{\text {ads }}=E_{\text {acene } / \mathrm{Au}(111)}-\left(E_{\mathrm{Au}(111)}+E_{\text {acene }}\right)
$$

where $E_{\mathrm{acene} / \mathrm{Au}(111)}$ is the total energy of the molecule/surface system, $E_{\mathrm{Au}(111)}$ is the energy of the bare $\mathrm{Au}(111)$ slab, and $E_{\text {acene }}$ is the energy of the isolated acene molecule in the gas phase.

Several approximations have been employed in our calculations to understand the energy trends for adsorption of acenes on $\mathrm{Au}(111)$. First, we used static unrelaxed molecular geometries for acenes. This approximation was assessed for the benzene/ $\mathrm{Au}(111)$ previously, ${ }^{35}$ finding a negligible impact of $0.02 \mathrm{eV}$ in the adsorption energy. Second, at the desorption temperatures in the low coverage limit the molecules are mobile and hence there is no unique adsorption site. Thus, the cleanest comparison of experiments and calculations at low coverage is done by assuming an unreconstructed $\mathrm{Au}(111)$ surface.

\section{ASSOCIATED CONTENT}

\section{Supporting Information}

The Supporting Information is available free of charge on the ACS Publications website at DOI: 10.1021/acs.jpclett.9b00265.

Sample preparation conditions, temperature-programmed desorption data, coverage dependent binding energies and pre-exponential factors of naphthaline, anthracene, and tetracene adsorbed on $\mathrm{Au}(111)$ (PDF)

\section{AUTHOR INFORMATION}

\section{Corresponding Author}

*E-mail: tegeder@uni-heidelberg.de. Phone: +49 (0) 622154 8475.

ORCID

Fairoja Cheenicode Kabeer: 0000-0003-1450-0615

Alexandre Tkatchenko: 0000-0002-1012-4854

Petra Tegeder: 0000-0002-5071-9385

Notes

The authors declare no competing financial interest.

\section{ACKNOWLEDGMENTS}

Funding by the German Research Foundation (DFG) through the collaborative research center SFB 1249 (Project B06) is gratefully acknowledged. A.T. received financial support from the European Research Council (ERC-CoG BeStMo).

\section{REFERENCES}

(1) Ihm, H.; Ajo, H. M.; Gottfried, J. M.; Bera, P.; Campbell, C. T. Calorimetric measurement of the heat of adsorption of benzene on Pt(111). J. Phys. Chem. B 2004, 108, 14627-14633.

(2) Morin, C.; Simon, D.; Sautet, P. Trends in the chemisorption of aromatic molecules on a $\mathrm{Pt}(111)$ surface: benzene, naphthalene, and anthracene from first principles calculations. J. Phys. Chem. B 2004, 108, 12084-12091.

(3) Gottfried, J. M.; Vestergaard, E. K.; Bera, P.; Campbell, C. T. Heat of adsorption of naphthalene on $\mathrm{Pt}(111)$ measured by adsorption calorimetry. J. Phys. Chem. B 2006, 110, 17539-17545.

(4) Silbaugh, T. L.; Campbell, C. T. Energies of formation reactions measured for adsorbates on late transition metal surfaces. J. Phys. Chem. C 2016, 120, 25161-25172.

(5) Carey, S. J.; Zhao, W.; Campbell, C. T. Energetics of adsorbed benzene on $\mathrm{Ni}(111)$ and $\mathrm{Pt}(111)$ by calorimetry. Surf. Sci. 2018, 676, 9-16.

(6) Fichthorn, K. A.; Miron, R. A. Thermal desorption of large molecules from solid surfaces. Phys. Rev. Lett. 2002, 89, 196103.

(7) Syomin, D.; Kim, J.; Koel, B. E.; Ellison, G. B. Identification of adsorbed phenyl (C6H5) groups on metal surfaces: Electron-induced dissociation of benzene on $\mathrm{Au}(111)$. J. Phys. Chem. B 2001, 105, 8387-8394.

(8) Liu, W.; Maaß, F.; Willenbockel, M.; Bronner, C.; Schulze, M.; Soubatch, S.; Tautz, F. S.; Tegeder, P.; Tkatchenko, A. Quantitative Prediction of Molecular Adsorption: Structure and Binding of Benzene on Coinage Metals. Phys. Rev. Lett. 2015, 115, 036104.

(9) Maass, F.; Jiang, Y.; Liu, W.; Tkatchenko, A.; Tegeder, P. Binding energies of benzene on coinage metal surfaces: Equal stability on different metals. J. Chem. Phys. 2018, 148, 214703.

(10) Lindstrom, C. D.; Muntwiler, M.; Zhu, X.-Y. Electron dynamics at polyacene $/ \mathrm{Au}(111)$ interfaces. J. Phys. Chem. B 2007, 111, 69136920.

(11) Gonella, G.; Dai, H.-L.; Rockey, T. J. Tetracene monolayer and multilayer thin films on $\operatorname{Ag}(111)$ : Substrate-adsorbate charge-transfer bonding and inter-adsorbate interaction. J. Phys. Chem. C 2008, 112, $4696-4703$ 
(12) Wheeler, W. D.; Parkinson, B. A.; Dahnovsky, Y. The adsorption energy and diffusion of a pentacene molecule on a gold surface. J. Chem. Phys. 2011, 135, 024702.

(13) Mete, E.; Danisman, M. F. Dispersion corrected DFT study of pentacene thin films on flat and vicinal $\mathrm{Au}(111)$ surfaces. J. Phys. Chem. C 2015, 119, 3596-3604.

(14) Redhead, P. A. Thermal desorption of gases. Vacuum 1962, 12, 203-211.

(15) Toyoda, K.; Hamada, I.; Lee, K.; Yanagisawa, S.; Morikawa, Y. Density functional theoretical study of pentacene/noble metal interfaces with van der waals corrections: vacuum level shifts and electronic structures. J. Chem. Phys. 2010, 132, 134703.

(16) Morbec, J. M.; Kratzer, P. The role of the van der Waals interactions in the adsorption of anthracene and pentacene on the $\mathrm{Ag}(111)$ surface. J. Chem. Phys. 2017, 146, 034702.

(17) King, D. A. Thermal desorption from metal surfaces: A review. Surf. Sci. 1975, 47, 384-402.

(18) Nieskens, D. L. S.; Van Bavel, A. P.; Niemantsverdriet, J. W. The analysis of temperature programmed desorption experiments of systems with lateral interactions; implications of the compensation effect. Surf. Sci. 2003, 546, 159-169.

(19) Tkatchenko, A.; DiStasio, R.; Car, R.; Scheffler, M. Accurate and efficient method for many-body van der Waals interactions. Phys. Rev. Lett. 2012, 108, 236402.

(20) Rockey, T. J.; Yang, M.; Dai, H.-L. Adsorption energies, interadsorbate interactions, and the two binding sites within monolayer benzene on Ag (111). J. Phys. Chem. B 2006, 110, 19973-19978.

(21) Schulze, M.; Bronner, C.; Tegeder, P. Adsorption energetics of azobenzenes on noble metal surfaces. J. Phys.: Condens. Matter 2014, 26,355004 .

(22) Hagen, S.; Leyssner, F.; Nandi, D.; Wolf, M.; Tegeder, P. Reversible Switching of tetra-tert-butyl-Azobenzene on a $\mathrm{Au}(111)$ Surface Induced by Light and Thermal Activation. Chem. Phys. Lett. 2007, 444, 85-90.

(23) Tegeder, P.; Hagen, S.; Leyssner, F.; Peters, M.; Hecht, S.; Klamroth, T.; Saalfrank, P.; Wolf, M. Electronic structure of the molecular switch tetra-tert-butyl-azobenzene adsorbed on $\operatorname{Ag}(111)$. Appl. Phys. A: Mater. Sci. Process. 2007, 88, 465-472.

(24) McNellis, E. R.; Bronner, C.; Meyer, J.; Weinelt, M.; Tegeder, P.; Reuter, K. Azobenzene versus tetra-tert-butyl-azobenzene (TBA) at $\mathrm{Au}(111)$ : characterizing the role of spacer groups. Phys. Chem. Chem. Phys. 2010, 12, 6404-6412.

(25) Bronner, C.; Priewisch, B.; Rück-Braun, K.; Tegeder, P. Potoisomerization of an azobenzene on the $\mathrm{Bi}(111)$ surface. J. Phys. Chem. C 2013, 117, 27031-27038.

(26) Óvári, L.; Luo, Y.; Leyssner, F.; Haag, R.; Wolf, M.; Tegeder, P. Adsorption and switching properties of a N-benzylideneaniline based molecular switch on a $\mathrm{Au}(111)$ surface. J. Chem. Phys. 2010, 133, 044707.

(27) Bronner, C.; Tegeder, P. Photo-induced and thermal reactions in thin films of an azobenzene derivative on $\mathrm{Bi}(111)$. New J. Phys. 2014, 16, 053004.

(28) Ibach, H.; Erley, W.; Wagner, H. The preexponential factor in desorption of CO on Ni(111). Surf. Sci. 1980, 92, 29-42.

(29) Mercurio, G.; McNellis, E. R.; Martin, I.; Hagen, S.; Leyssner, F.; Soubatch, S.; Meyer, J.; Wolf, M.; Tegeder, P.; Tautz, F. S.; Reuter, $\mathrm{K}$. Structure and energetics of azobenzene on $\mathrm{Ag}(111)$ : benchmarking semiempirical dispersion correction approaches. Phys. Rev. Lett. 2010, 104, 036102.

(30) Bronner, C.; Schulze, M.; Hagen, S.; Tegeder, P. The influence of the electronic structure of adsorbate-substrate complexes on photoisomerization ability. New J. Phys. 2012, 14, 043023.

(31) Mercurio, G.; Maurer, R.; Liu, W.; Hagen, S.; Leyssner, F.; Tegeder, P.; Meyer, J.; Tkatchenko, A.; Soubatch, S.; Reuter, K.; Tautz, F. Quantification of finite-temperature effects on adsorption geometries of p-conjugated molecules: Azobenzene/Ag(111). Phys. Rev. B: Condens. Matter Mater. Phys. 2013, 88, 035421.

(32) Ruiz, V. G.; Liu, W.; Zojer, E.; Scheffler, M.; Tkatchenko, A. Density-Functional Theory with Screened van der Waals Interactions for the Modeling of Hybrid Inorganic-Organic Systems. Phys. Rev. Lett. 2012, 108, 146103.

(33) Maurer, R. J.; Ruiz, V. G.; Tkatchenko, A. Many-Body Dispersion Effects in the Binding of Adsorbates on Metal Surfaces. J. Chem. Phys. 2015, 143, 102808.

(34) Hermann, J.; DiStasio, R. A., Jr; Tkatchenko, A. First-principles models for van der Waals interactions in molecules and materials: Concepts, theory, and applications. Chem. Rev. 2017, 117, 4714.

(35) Carrasco, J.; Liu, W.; Michaelides, A.; Tkatchenko, A. Insight into the description of van der Waals forces for benzene adsorption on transition metal (111) surfaces. J. Chem. Phys. 2014, 140, 084704.

(36) Blum, V.; Gehrke, R.; Hanke, F.; Havu, P.; Havu, V.; Ren, X.; Reuter, K.; Scheffler, M. Ab initio molecular simulations with numeric atom-centered orbitals. Comput. Phys. Commun. 2009, 180, 2175.

(37) Havu, V.; Blum, V.; Havu, P.; Scheffler, M. Efficient $\mathrm{O}(\mathrm{N})$ integration for all-electron electronic structure calculation using numeric basis functions. J. Comput. Phys. 2009, 228, 8367.

(38) Perdew, J. P.; Burke, K.; Ernzerhof, M. Generalized gradient approximation made simple. Phys. Rev. Lett. 1996, 77, 3865.

(39) van Lenthe, J. H.; Faas, S.; Snijders, J. G. Gradients in the ab initio scalar zeroth-order regular approximation (ZORA) approach. Chem. Phys. Lett. 2000, 328, 107-112.

(40) Ruiz, V. G.; Liu, W.; Tkatchenko, A. Density-Functional Theory with Screened van der Waals Interactions Applied to Atomic and Molecular Adsorbates on Close-Packed and Non-Close-Packed Surfaces. Phys. Rev. B: Condens. Matter Mater. Phys. 2016, 93, 035118. 